Roquette, M.E.T.; Pavan-Filho, F.; Costa, T.J.F.; Carvalho, R.C. Projeto Monitores Marinhos (MOMAR): proporcionando uma nova visão dos ambientes marinhos capixabas. Revista Brasileira de Ecoturismo, São Paulo, v.3, n.2, 2010, pp.273-282.

\title{
Projeto Monitores Marinhos (MOMAR): proporcionando uma nova visão dos ambientes marinhos capixabas
}

\author{
Maria Elisa Tosi Roquette, Flavio Pavan Filho, \\ Thiago José Fagundes e Costa, Renata Costa Carvalho
}

\section{RESUMO}

Com o aumento populacional, turístico e econômico ao longo do litoral brasileiro, cada vez mais é necessária a realização de levantamentos e monitoramentos de comunidades biológicas e seus fatores governantes, bióticos ou abióticos, pois estes estudos auxiliam o entendimento de como os fatores antrópicos podem interferir nas comunidades biológicas. A preservação dos ambientes recifais se torna cada vez mais necessária diante a problemática encontrada em nossos sistemas marinhos. O primeiro passo em direção a preservação é o conhecimento acerca do que se pretende preservar. O conhecimento é fundamental para que se estabeleçam ações visando à educação ambiental. Conhecendo os ambientes marinhos é possível apresentar às pessoas a verdadeira importância ecológica e turística dos oceanos. Iniciativas de monitoramento marinho vêm sendo desenvolvidas em projetos como o Reef Check, Coral Vivo, entre outros, buscando a compreensão dos sistemas recifais. O projeto MOMAR é mais uma iniciativa com o objetivo de aplicar metodologias novas e não destrutivas que busca a formação de uma base de dados que auxilie a compreensão e conservação dos ambientes marinhos do estado do Espírito Santo, área de alta prioridade para conservação e que não conta com iniciativas de monitoramento. O projeto possui o intuito de estabelecer monitoramento em longo prazo, conhecendo e divulgando a realidade de nossos ambientes marinhos para a sociedade, além de fornecer subsídios aos gestores ambientais para o manejo de sistemas recifais e oportunidades de estudos científicos para estudantes e pesquisadores do estado. A divulgação dos resultados ocorrerá por meio de cartilhas, palestras em faculdades, escolas, congressos e simpósios, além da produção de material para distribuição em escolas da rede pública e privada. Com isso, pretendemos sensibilizar e conscientizar turistas, praticantes de pesca e caça submarina e de mergulho autônomo para um maior conhecimento dos organismos encontrados durante a prática. As campanhas para a coleta de dados serão realizadas utilizando metodologias de censo visual e de fotoquadrate. Os dados coletados serão colocados em planilha padrão para análises, confecção de trabalhos científicos e divulgação de dados para a sociedade em geral. Os dados proporcionarão a confecção de guias de identificação de peixes e bentos, que apresentarão informações sobre a situação de ameaça (IUCN), categoria trófica e ecologia. A efetivação do projeto MOMAR permitirá a criação de um registro permanente da diversidade. $O$ monitoramento constante de áreas permitirá a constatação do estado de conservação e ajudará a garantir a proteção, manutenção e o conhecimento da vida marinha.

PALAVRAS-CHAVE: Ambientes Marinhos, Monitoramento, Conservação. 


\section{Marine Monitors Project (MOMAR): providing a new view of marine environments of Espírito Santo state, southeastern Brazil}

\section{ABSTRACT}

With population, tourism and economic development increase along the Brazilian coast, is increasingly necessary to carry out surveys and monitoring of biological communities and their governing factors, biotic or abiotic, since these studies aid the understanding of how anthropogenic factors may interfer biological communities. The preservation of reef environments becomes increasingly necessary behind the problems found in our marine systems. The first step toward preserving is the knowledge about what is to be preserved. Knowledge is essential to establish actions aimed at environmental education. Knowing the marine environment it is possible to provide people the true ecological importance of the oceans and tourism. Marine monitoring initiatives have been developed in projects such as Reef Check, Coral Vivo, among others, seeking the understanding of reef systems. MOMAR Project is an initiative aiming to implement new and improved non-destructive metodologies that pursues the development of a database to assist the understanding and conservation of marine environments of the Espirito Santo State, an area of high priority for conservation and has not monitoring initiatives. The project has the aim of establishing long-term monitoring, disseminating the reality of our marine environment for society, and provides subsidies to environmental managers for the management of reef systems and scientific research opportunities for students and researchers in the state. Dissemination will occur through pamphlets, lectures at colleges, schools, conferences and symposia, in addition to producing material for distribution in public and private schools. We intend to raise awareness and educate tourists, anglers and spear fishing and scuba diving to a greater knowledge of organisms encountered in practice. The collection of data campaigns will be performed using visual census methodologies and Photoquadrat. The collected data will be placed in standard spreadsheet for analysis, preparation of scientific papers and dissemination of results to society in general. The data will provide the preparation of guides to the identification of fish and benthos, which will present information on the situation of threat (IUCN), the trophic categories and ecology. The effectiveness of the MOMAR Project will create a permanent record of diversity. Constant monitoring of areas allows the observation of the condition and helps to ensure the protection, maintenance and knowledge of marine life.

KEYWORDS: Marine Environment, Monitoring, Conservation. 
Roquette, M.E.T.; Pavan-Filho, F.; Costa, T.J.F.; Carvalho, R.C..

\section{Introdução}

O aumento populacional, turístico e econômico ao longo das zonas costeiras de todo o mundo tem elevado a preocupação em relação ao uso dos recursos e preservação das belezas cênicas (SOBHEE, 2004; PINHEIRO et al., 2009). No litoral do Brasil, tanto as atividades de recreação como a pesca possuem enorme importância social e econômica, contudo, encontram-se amplamente desordenadas (TAKAHASHI, 2004; ISAAC et al., 2006). Atividades insustentáveis, como destinação inadequada de resíduos, lançamento de efluentes em corpos d'água, ocupação desordenada da região litorânea, supressão da vegetação costeira, são praticadas naturalmente sem que nem haja consciência pelas ações praticadas.

Uma mudança de paradigma pela sociedade conta com o levantamento e a disseminação de informações sobre o meio ambiente em que estamos inseridos e a reflexão sobre essas atividades. Neste ínterim, existe uma demanda para a realização de levantamentos sistematizados de comunidades biológicas e dos fatores bióticos ou abióticos que às influenciam, pois estes dados auxiliam no entendimento de como a antropização pode interferir no ambiente natural. Além disso, conhecer os ambientes marinhos possibilita às pessoas demonstrar à importância ecológica e turística dos sítios oceânicos. Iniciativas de monitoramento marinho vêm sendo desenvolvidas em projetos como o Reef Check, Coral Vivo, Instituto Laje Viva entre outros, buscando a compreensão dos sistemas recifais.

Destacam-se no estado do Espírito Santo muitas atividades turísticas e recreacionais, como o mergulho e a pesca, que são focadas nos ambientes costeiros. Apesar dos impactos inevitáveis de cada atividade ao meio ambiente, devido ao uso e exploração dos recursos (MAGRO, 2001), é possível minimizá-los com gestão e educação, aumentando a qualidade da experiência do praticante e, consequentemente, sua disposição em conservar (MORAES, 1999). Iniciativas que envolvem educação ambiental em ambientes marinhos desenvolvidas em Ubatuba, SP, através do snorkeling e mergulho autônomo, (BERCHEZ et al., 2007), se mostram eficientes, pois proporcionam ao turista um acréscimo significativo às interrelações entre os compartimentos socioambientais (PEDRNI et al., 2009).

O estado do Espírito Santo possui uma rica diversidade marinha (FLOETER; GASPARINI, 2000; PEREIRA; GUIMARAES, 2002) e aparece como área de alta prioridade para conservação (BRASIL, 2007). Contudo, mesmo com o aumento da prática de atividades que permitem o contato direto com a natureza (TOLEDO, 2002), o estado ainda carece de projetos que visam o ordenamento das distintas formas de uso e a disseminação de uma abordagem ecológica às atividades. O Projeto MOMAR, criado em 2009 no Espírito Santo, visa o preenchimento de lacunas a respeito de monitoramento e conscientização para o uso sustentável dos ambientes costeiros capixabas. Este trabalho tem o objetivo de descrever as principais metas, desafios e resultados do projeto no Espírito Santo. 


\section{Propósitos a serem atingidos pelo MOMAR}

O Projeto MOMAR é uma iniciativa criada por uma parceria entre uma operadora de mergulho e uma organização não governamental sem fins lucrativos, que ministram cursos de extensão de mergulho científico para alunos de biologia de universidades e pessoas de áreas afins. A utilização da atividade de mergulho autônomo com métodos científicos não destrutivos tem se mostrado um instrumento de importância fundamental na realização de trabalhos de campo em ambientes aquáticos, gerando informações importantes para caracterização do meio ambiente marinho e monitoramento.

Buscando conhecer a realidade dos ecossistemas marinhos do Espírito Santo, bem como seus estados de conservação, o projeto tem como objetivo realizar levantamentos e estudos biológicos dos ambientes recifais capixabas a fim de se estabelecer um monitoramento à longo prazo dos mesmos.

O banco de dados gerados através dos estudos servirá como ferramentas para subsidiar o manejo de sistemas recifais por parte dos gestores ambientais, bem como proporcionar oportunidades de estudos científicos não destrutivos para estudantes e pesquisadores do estado do Espírito Santo.

Além disso, o projeto objetiva atingir à sociedade em geral ao divulgar o conhecimento adquirido, de forma a sensibilizá-la e promover uma conscientização acerca dos problemas ambientais. $O$ projeto visa proporcionar àqueles que já possuem um maior contato com esses ecossistemas, turistas em geral, praticantes de mergulho e pesca, a oportunidade de desenvolverem uma nova visão sobre o ambiente marinho e recursos explorados.

\section{Justificativa}

A região costeira do Espírito Santo é habitada por um grande número de espécies tropicais e subtropicais (GASPARINI et al., 2000), possuindo uma alta diversidade de peixes (FLOETER; GASPARINI, 2000), organismos bentônicos (PEREIRA; GUIMARAES, 2002; PEREIRA FILHO, 2008) bem como a ocorrência de quelônios (MARCOVALDI; MARCOVALDI, 1999) e mamíferos marinhos (FREITAS NETTO; BARBOSA, 2003). Apesar de sua importância, o litoral tem sofrido com o turismo, urbanização e industrialização desordenadas, o que pode gerar grandes impactos para as comunidades naturais (SOBHEE, 2004). Como foi preconizado por Nixon (1995), o aumento populacional, por si só, não somente impacta a qualidade das águas costeiras, como também aumenta a pressão sobre a pesca, a destruição de habitats e a descarga de resíduos industriais.

Programas de monitoramento ambiental, através do diagnóstico das comunidades bentônicas e de peixes, são importantes para avaliação dos impactos e sustentabilidade das atividades humanas (SABINO; ANDRADE, 2003; FLUCKIGER, 2006) e podem auxiliar na gestão dos ecossistemas. A falta de registros históricos e estudos sazonais encorajam o Projeto MOMAR a um monitoramento a longo prazo, inicialmente 
com uma abrangência local (Vitória e Vila Velha), partindo para regional (ES).

As técnicas não destrutivas utilizadas pelo projeto possuem grandes vantagens quando comparadas aos métodos tradicionais de coleta de dados. A captura de imagens no campo apresenta um aumento da eficácia da amostragem e da extensão da área amostrada, mantendo uma boa resolução taxonômica, além do registro histórico permanente que pode ser utilizado para futuras comparações com novas amostras. Os censos visuais da comunidade de peixes permitem uma avaliação ecológica rápida, com um imediato processamento das amostras, permitindo uma eficiente descrição dos ecossistemas.

A falta de informações ecológicas por parte da população humana faz com que atitudes insustentáveis sejam praticadas cotidianamente. Uma nova visão dos ambientes marinhos e recursos naturais é necessária para se alcançar uma relação mais harmônica com o meio em que vivemos.

\section{Metodologia}

\section{Áreas de Monitoramento}

Inicialmente o projeto abrange os ambientes recifais dos municípios de Vitória e Vila Velha: Ilha do Boi (Gaetas) e Ilha dos Pacotes, respectivamente (Figura 1). A ilha dos Pacotes situa-se a $2 \mathrm{~km}$ da linha de costa e Ilha da Gaeta situa-se a 300 metros da costa. Ambas as ilhas são afloramentos rochosos e seus recifes atingem até 15 metros de profundidade.

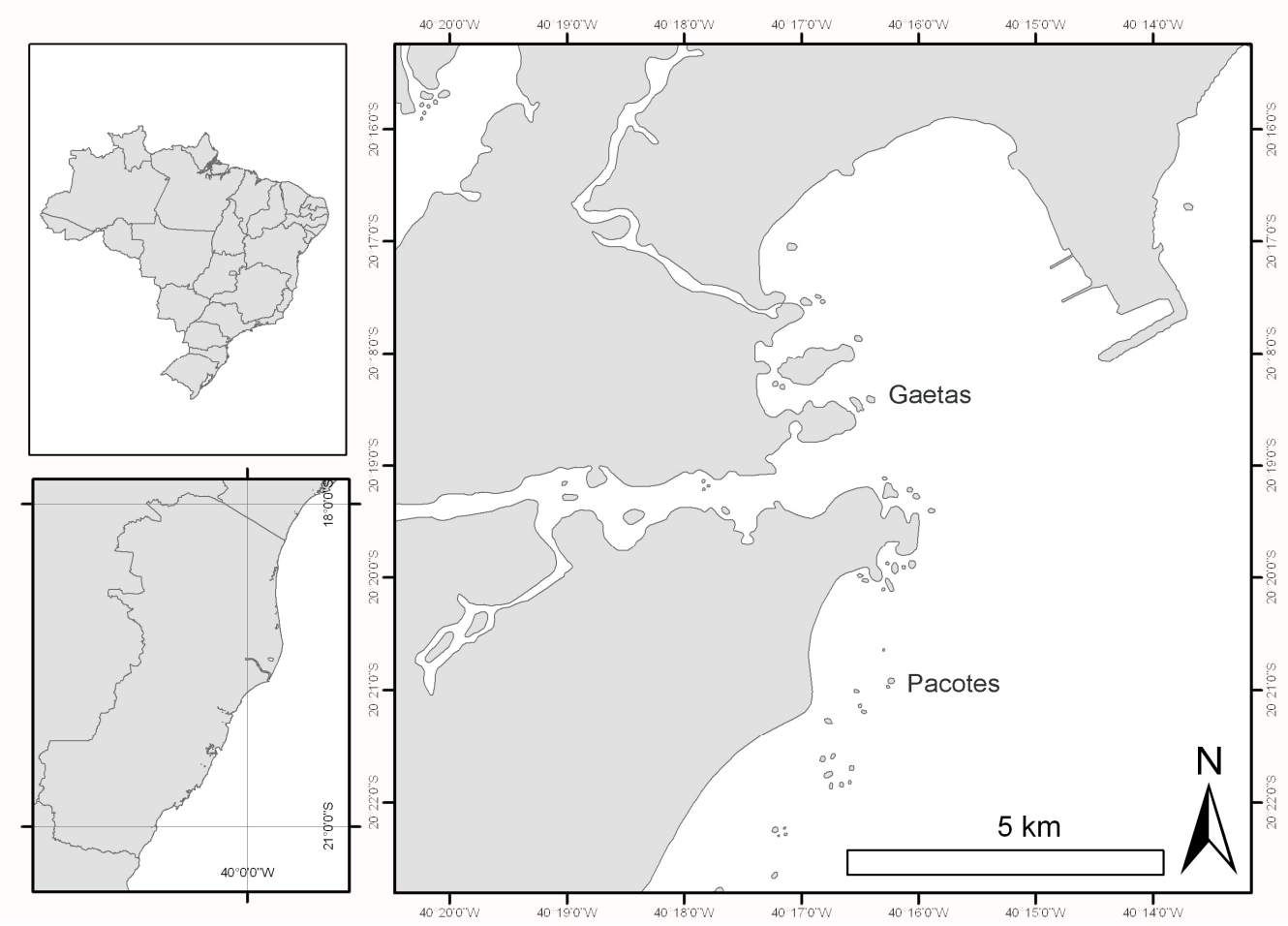

Figura 1. Área de estudo mostrando os municípios de Vitória e Vila Velha com as respectivas ilhas estudadas. 


\section{Coleta de dados}

Censo visual: a amostragem quantitativa é feita por meio de censos visuais através da utilização do mergulho autônomo, no qual são contadas as espécies de peixes e indivíduos presentes em um transect de $20 \mathrm{~m} \times 2 \mathrm{~m}$ (método denominado "Strip Transect" - Kingsford \& Battershil, 2000 - Figura 2A). A contagem é realizada em classes de tamanho: 0-10 cm; $11-20 \mathrm{~cm} ; 21-30 \mathrm{com}$ e $>40 \mathrm{~cm}$. Além disso, variáveis ambientais como profundidade e complexidade estrutural do fundo (alta, média e baixa) são registradas.

Foto-quadrat: a amostragem da comunidade bentônica (percentual de cobertura) do infralitoral é realizada através da utilização do mergulho autônomo em duplas, utilizando-se um transect com o comprimento do recife rochoso, paralelo ao costão onde um registro fotográfico é feito a cada 1 metro de deslocamento (Figura 2B). O equipamento utilizado é feito por uma armação de PVC e máquina digital acoplada, formando um equipamento único. A análise de dados será feita através da metodologia de contagem de pontos escolhidos aleatoriamente nos quadrats, com auxílio do software CPCe v. 3.6 (Coral Point with Excel Extensions). Esse programa gera aleatoriamente o número de pontos determinados pelo pesquisador, que localiza e identifica os pontos sorteados por sobreposição, e calcula o percentual de cobertura da espécie identificada, a média de cobertura, desvio padrão, erro padrão e o índice diversidade ShannonWeaver de cada localidade.

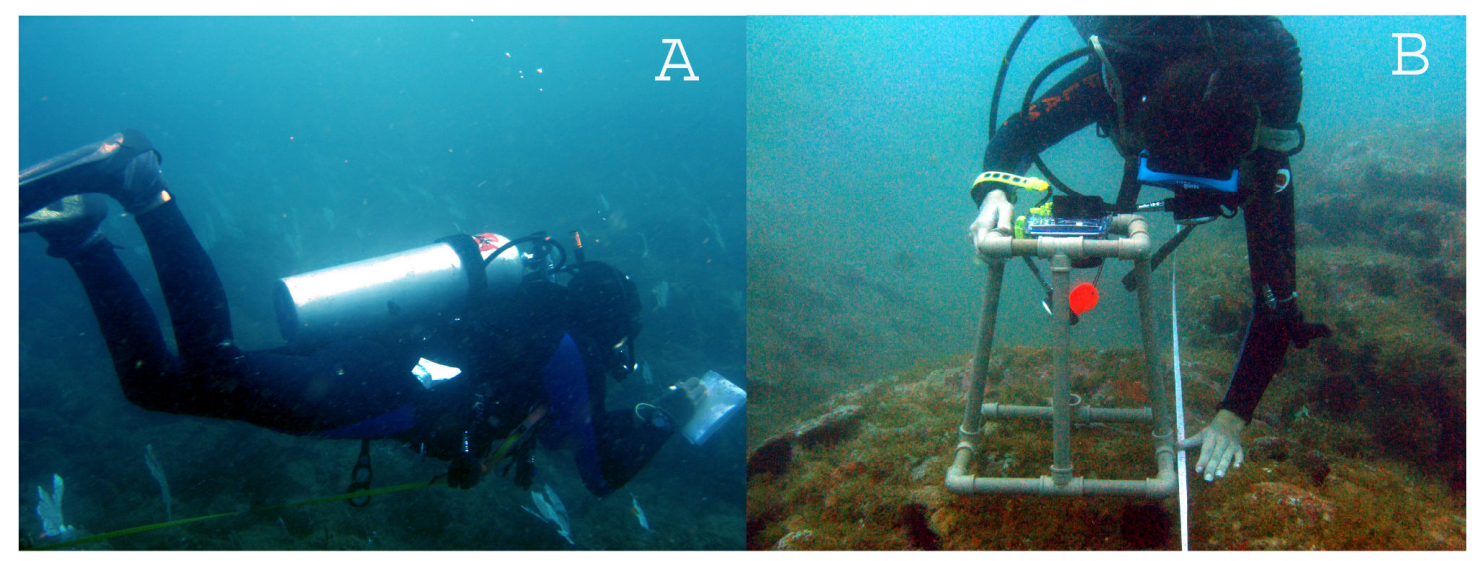

Figura 2. Metodologias utilizadas nos trabalhos, censos visuais das comunidades de peixes $(A)$ e registro fotográfico das comunidades de bentos $(B)$.

\section{Interação com a sociedade}

Atividades de mergulho praticadas por todas as operadoras e empresas de mergulho apenas se preocupam em propiciar um mergulho seguro para os pratican- 
tes. Nesta primeira etapa do Projeto, praticantes de mergulho estão tendo a oportunidade de interagir de forma diferente com o ambiente marinho recifal, onde são capacitados a reconhecer e estudar novos organismos aquáticos. Esta atividade tem propiciado ao praticante compreender a importância das espécies no ambiente natural e diagnosticar o estado de conservação local, através da presença ou ausência de espécies ameaçadas ou importantes economicamente.

As próximas atividades do Projeto MOMAR envolvem a divulgação dos resultados para a comunidade científica e comunidade capixaba em geral. Serão elaborados artigos científicos e palestras nas universidades visando a divulgação no meio acadêmico. Informações gerais do ambiente costeiro serão apresentadas nas escolas e associações comunitárias a fim de se ressaltar a importância da conservação e do conhecimento marinho.

Os resultados obtidos são ferramentas importantes para a gestão ambiental, uma vez que serão levantados o conhecimento das comunidades faunísticas e a análise sazonal das áreas amostradas, aumentando o entendimento sobre a dinâmica das populações.

Os recursos marinhos são finitos e os ambientes aquáticos estão sendo massivamente modificados pela ação antrópica com consequências incalculáveis. Esperamos que a divulgação desses dados possa mudar o pensamento acerca do ambiente costeiro para a sociedade em geral.

\section{Resultados preliminares}

Concernentes ao censo visual foram identificadas espécies pertencentes às famílias Acanthuridae, Chaetodontidae, Pomacentridae, Haemulidae, Pomacanthidae, Tetraodontidae, Sciaenidae, Sparidae, Mullidae, Monacanthidae, Holocentridae, Labridae, Scaridae e Ophichthidae, com destaque para as espécies Acanthurus bahianus, Acanthurus chirurgus, Halichoeres poeyi, Chromis multilineata e Chaetodon striatus, como as mais abundantes.

Vale salientar que em uma das áreas estudadas, llha dos Pacotes, é recorrente a observação da espécie Gramma brasiliensis, que se encontra ameaçada de extinção (BRASIL, 2008).

A comunidade bentônica observada compreende organismos importantes à ecologia marinha, como os formadores de estruturas coralinas, bem como organismos ameaçados comercialmente e com risco de extinção.

As espécies zoobentônicas observadas foram os octocorais Carijoa riisei, Heterogorgia uatumani, Muriceopsis sulphurea, Phyllogorgia dilatata, Plexaurella grandiflora; os hexacorais Actinoporus elegans, Palythoa caribaeorum, Siderastrea stellata, Zoanthus sociatus, Favia gravida; o coral fogo Millepora alcicornis, o crinóide Tropiometra carinata; Gorgonocéfalo "estrela-cesto"; o briozoário Schizoporella unicornis; os ouriços Echinometra lucunter, Lytechinus variegatus, Eucidaris tribuloides; Esponjas; Hidrozoários e Ascídias. Também foi observada uma grande quantidade de espécies 
fitobentônicas, organismos fundamentais ao ecossistema marinho, por serem produtores primários de energia, com destaque para algas calcárias incrustantes e articuladas, assim como a Chlorophyta Halimeda sp., espécies com grande importância na formação de recifes de corais, por apresentarem grandes quantidades de substância calcária em suas composições

\section{Perspectivas}

Através do presente estudo são feitos levantamentos dos organismos que compõem a comunidade recifal de ilhas costeiras capixabas, além da descrição da estrutura das comunidades de peixes e organismos bentônicos de tais ambientes. Para analisar a influência de fatores antrópicos e/ou ambientais sobre esses sistemas serão feitas análises temporais dessas comunidades.

Todos os resultados dos levantamentos e monitoramento serão trabalhados junto aos órgãos ambientais municipais e estaduais, para que as tomadas de decisão sejam efetivas e os benefícios para a sociedade sejam catalisados.

Para atingir a comunidade, como turistas, admiradores, estudantes, praticantes de caça e pesca, serão confeccionados materiais informativos e desenvolvidas atividades interativas de fácil compreensão acerca da ecologia da fauna marinha, bem como sua conservação. Dessa forma, será possível proporcionar trocas de conhecimento, proporcionando-os uma visão mais ecológica do ambiente, enquanto os mesmos praticam suas atividades de recreação, além de incentivá-los a participar na ajuda à preservação e conservação desses ambientes.

\section{Referências Bibliográficas}

BERCHEZ, F.; GHILARDI, N.; ROBIM, M. J.; PEDRINI, A. G.; HADEL, V. F.; FLUKIGER, G.; SIMÕES M.; MAZZARO, R.; KLAUSENER, C.; SANCHES, C.; BESPALEC, $P$. Projeto trilha subaquática: sugestão de diretrizes para a criação de modelos de educação ambiental em unidades de conservação ligadas a ecossistemas marinhos. Revista OLAM - Ciência e Tecnologia. Rio Claro, 2007. $n^{\circ}$. 3, v.7, p.181-209

BRASIL.MMA. Brazilian protected areas. Brasília: Ministry of Environment. 2007.

BRASIL. MMA. Livro vermelho da fauna brasileira ameaçada de extinção. Brasília:MMA, 2008.

FLOETER, S.R.; GASPARINI, J.L. The southwestern Atlantic reef fish fauna: composition and zoogeographic patterns. Journal of Fish Biology. 2000. v.56, p. 1099-1114.

FLUCKIGER, G. Levantamento fisionômico da comunidade bentônica de um trecho do costão rochoso sul da Praia das Toninhas, Ubatuba-SP (Brasil). Tese (Bacharelado). Instituto de oceanografia. Universidade de São Paulo. São Paulo, 2006. 45p. 
FREITAS NETTO, R.; BARBOSA, L.A. Cetacean and fishery interactions along the Espirito Santo State, Southeastern Brazil during 1994-2001. The Latin American Journal of Aquatic Mammals. 2003. $n^{\circ} .2$, v.1, p.57-60.

GASPARINI, J. L.; FLOETER, S. R.; GANDOLFI, S. M. Proposta para criação do Parque Estadual marinho llhas de Guarapari, Espírito Santo. In: Anais do V Simpósio de Ecossistemas Brasileiros: Conservação. Vitória, 2000.

ISAAC, V.J.; MARTINS, A.S.; HAIMOVICI, M; CASTELLO, J.P.; ANDRIGUETTO FILHO, J.M. Síntese do estado de conhecimento sobre a pesca marinha e estuarina do Brasil. In: ISAAC, V.J.; MARTINS, A.S.; HAIMOVICI, M.; ANDRIGUETTO FILHO, J.M. A pesca marinha e estuarina do Brasil no início do século XXI: recursos, tecnologias, aspectos socioeconômicos e institucionais. Universidade Federal do Pará.. Belém, 2006. p.181-186.

KINGSFORD M.J.; BATTERSHILL C.N. Subtidal habitats and benthic organisms of rocky reefs. In: M.J., KINGSFORD; C.N., BATTERSHILL. Studying temperate marine environments. A handbook for ecologists. Canterbury University Press \& CRC Press, Chistchurch. New Zealand, 2000. p.84-114.

MAGRO, T. C. Manejo do uso público em áreas naturais protegidas - aspectos naturais e sociais. São Paulo: Escola Superior de Agronomia Luiz de Queiroz. Universidade de São Paulo, 2001.

MARCOVALDI, M.A.; MARCOVALDI, G.G. Marine turtles of Brazil: the history and structure of Projeto TAMAR-IBAMA. Biology Conservation. 1999. $\mathrm{n}^{\circ}$. 91, p.35-41.

MORAES, A. C. R. Contribuições para a gestão da zona costeira do Brasil: elementos para uma geografia do litoral brasileiro. Hucitec./Edusp. São Paulo, 1999. $229 \mathrm{p}$.

NIXON, S. W. Coastal marine eutrophication: A definition, social causes, and future concerns. Ophelia. 1995. $n^{\circ} .41$, p.199-219.

PEDRINI, A. G.; ANDRADE-COSTA, E. S; SILVA, V. G; PINA, R.; SABA, M. G.; BERCHEZ, F. Projeto EcoTurisMar: uma proposta de educação/interpretação ambiental para o ecoturismo marinho em áreas protegidas. Estudo de caso preliminar no Parque Estadual da Ilha Anchieta, São Paulo, Brasil. In: Anais da XI Semana Nacional de Oceanografia. Belém, 2009.

PEREIRA. A.P.V. \& GUIMARÃES, S.M.P.B. Taxonomia e estrutura da comunidade bentônica da praia da Baleia - Manguinhos - Serra (ES). In: Anais da Reunião Brasileira de FicologiaA, 9. 2002, ES. Resumos. Santa Cruz , 2002. 217p.

PEREIRA FILHO, G. H. Caracterização das comunidades marinhas bentônicas de substrato consolidado da llha do Francês (ES) baseado em unidades da paisagem. Tese (Doutorado). Universidade de São Paulo. São Paulo, 2008. 198p. 
PINHEIRO, H. T.; FERREIRA, A. L.; MOLINA, R. P.; PROTTI, L. M. C.; ZANARDO, S. C.; JOYEUX, J-C; DOXSEY, J. R. Profile of social actors as a tool the definition of marine protected areas: the case of the llha dos Franceses, southern coast of Espírito Santo, Brazil. Technical - Scientific Articles. Natureza \& Conservação. 2009. v. 7, $\mathrm{n}^{\circ} .1, \mathrm{p} .181-194$.

SABINO, J.; ANDRADE, J.P. Uso e conservação da ictiofauna no ecoturismo da região de Bonito, Mato Grosso do Sul: O mito da sustentabilidade ecológica no rio Baía Bonita (Aquário Natural de Bonito). Biota Neotropica. 2003. v.3, n.2, p.1-9.

SOBHEE, S.K. Economic development income inequality and environmental degradation of fisheries resources in Mauritius. Environmental Management. 2004. v.34, $\mathrm{n}^{\circ} .1, \mathrm{p} .150-157$.

TAKAHASHI, L. Uso público em unidades de conservação. Cadernos de Conservação. 2004. v.2, n.2, p.1-40.

TOLEDO, R. F.. A educação ambiental em unidades de conservação do estado de São Paulo. Tese (Mestrado). Faculdade de Saúde Publica. Universidade de São Paulo. São Paulo, 2002.

Maria Elisa Tosi Roquette: Projeto Monitores Marinhos.

Email: mariaelisa_me@hotmail.com

Link para o Lattes: http://lattes.cnpq.br/7440396482864685

Flavio Pavan Filho: Projeto Monitores Marinhos.

Email: flaviodive@yahoo.com.br

Thiago José Fagundes e Costa: Projeto Monitores Marinhos.

Email: thiagojfc@hotmail.com

Link para o Lattes: http://lattes.cnpq.br/2489670930142889

Renata Costa Carvalho: Projeto Monitores Marinhos.

Email: recosca@yahoo.com.br

Link para o Lattes: http://lattes.cnpq.br/4288259208337133

Data de submissão: 03 de novembro de 2009.

Data do aceite: 12 de abril de 2010 . 